\title{
Los comités hospitalarios de bioética y la educación en salud: notas para la discusión"
}

\section{Hospital bioethics and health education committees: Notes for discussion}

\section{As comissões hospitalares de bioética e a educação em saúde: notas para a discussão}

Fecha de recepción: 10 de septiembre de 2016

Fecha de evaluación: 15 de noviembre de 2016

Fecha de aceptación: 30 de noviembre de 2016

Disponible en línea: 1 de diciembre de 2016

Jorge Alberto Álvarez**

Sergio López Moreno ${ }^{* * *}$

DoI: http://dx.doi.org/10.18359/rlbi.2343

Cómo citar:

Álvarez, J. A. y López Moreno, S. (2016). Los comités hospitalarios de bioética y la educación en salud: notas para la discusión. Revista Latinoamericana de Bioética, 17(1), 184-199. DoI: http://dx.doi.org/10.18359/rlbi.2343

Trabajo preparado para la 8a Reunión Nacional de Comisiones Estatales de Bioética de la Comisión Nacional de Bioética de México; presentado como conferencia. No se ha publicado total ni parcialmente.

** Doctor en ciencias sociosanitarias y humanidades médicas. Profesor investigador (titular C) del Departamento de Atención a la Salud de la Universidad Autónoma Metropolitana Unidad Xochimilco. Correo electrónico: jalvarez@correo.xoc.uam.mx. ORCID: http://orcid.org/00000001-9935-8632. Ciudad de México, México.

*** Doctor en epidemiología. Profesor investigador (titular C) del Departamento de Atención a la Salud de la Universidad Autónoma Metropolitana Unidad Xochimilco. Correo electrónico institucional: slopez@correo.xoc.uam.mx. ORCID: http://orcid.org/0000-0002-7543-1783.Ciudad de México, México. 


\title{
Resumen
}

Este trabajo toma el caso mexicano de la exigencia legal de comités de bioética: comités hospitalarios de bioética ([CHB], comités de ética asistencial o comités de ética clínica) y los comités de ética en investigación (CEI); la Ley General de Salud hace obligatorios esos comités desde una modificación en 2011. La literatura refiere que los comités de bioética cuentan con tres funciones básicas: educativa, consultiva y normativa; por su parte el marco legal vigente retoma elementos teóricos importantes, uno de ellos corresponde a las funciones de los comités de bioética. Adicionalmente, profundiza en el análisis de la función educativa, toda vez que una buena parte de la literatura disponible se dirige a la función consultiva. Asimismo, se realizan algunas propuestas sobre contenidos en materia educativa. Así pues, es posible organizar grupos de problemas éticos en torno al inicio de la vida humana y problemas éticos alrededor del final de la vida humana. Efectivamente, hay muchos problemas éticos toda la vida, pero entrar o salir de la vida parece ser más conflictivo desde el punto de vista ético.

Palabras clave: comités de bioética, educación en bioética, eutanasia, interrupción voluntaria del embarazo.

\begin{abstract}
This paper takes the Mexican case of the legal requirement of bioethics committees: hospital bioethics committees (CHB; healthcare ethics committees or clinical ethics committees), and ethics committees in research (CEI). The General Health Law makes these committees compulsory since a modification in 2011. The literature says that bioethics committees have at least three basic functions: educational, advisory and normative. For its part, the current legal framework takes up important theoretical elements. One of which corresponds to the functions of bioethics committees. Also, it delves into the analysis of the educational function, since much of the available literature addresses the advisory role. Likewise, some proposals are made regarding contents in educational matters. Thus it is possible to organize groups of ethical problems around the beginning of human life and ethical problems around the end of human life. Indeed, there are many ethical problems along life, but getting in or out of life seems to be more controversial from the ethical point of view.
\end{abstract}

Keywords: bioethics committees, education in bioethics, euthanasia, voluntary termination of pregnancy.

\section{Resumo}

O presente trabalho pega o caso mexicano da exigência legal de comissões de bioética: comissões hospitalares de bioética (CHB; comissões de ética assistencial ou comissões de ética clinica) e os comissões de ética em investigação (CEI); a Lei Geral da Saúde torna obrigatórios estas comissões a partir de uma modificação em 2011. A literatura refere que as comissões de bioética têm três funções básicas: educativa, consultiva e de regulamentos; por sua vez, o quadro jurídico em vigor retoma elementos teóricos importantes, um deles corresponde às funções das comissões de bioética. Além disso, aprofunda na análise da função educativa, toda vez que uma grande parte da literatura disponível dirige-se à função consultiva. Além disso, realizam-se algumas propostas sobre os conteúdos referentes à educação. Assim então, é possível organizar grupos de questões éticas em torno a o início da vida humana e questões éticas em torno a o fim da vida humana. Na verdade, existem muitos problemas éticos a vida toda, mas entrar ou sair da vida parece ser mais controverso do ponto de vista ético.

Palavras-chave: comissões de bioética, educação em bioética, eutanásia, interrupção voluntaria do embaraço. 


\section{Introducción}

El 14 noviembre de 2011, el Diario Oficial de la Federación (DOF) de México publicó un decreto (DOF, 2011) que reformó los artículos 41 bis y 98 de la Ley General de Salud, estableciendo la obligación de todos los establecimientos de atención del Sistema Nacional de Salud de integrar -según su grado de complejidad y nivel de resolución- un Comité Hospitalario de Bioética (CHB, nombre dado a los Comités de Ética Asistencial) y un Comité en Ética de la Investigación (CEI). De acuerdo con el decreto, los CHB deben establecerse de manera obligatoria en todos los establecimientos del sector público, social y privado, con el fin de apoyar la toma de decisiones respecto a los problemas bioéticos que se presenten en la práctica clínica o en la docencia que se imparte en el área de salud.

El 31 de octubre de 2012, el DoF publicó el Acuerdo que establece las Disposiciones Generales para la Integración y Funcionamiento de los Comités Hospitalarios de Bioética (Diario Oficial de la Federación, 2012), incorporando en gran medida los criterios elaborados previamente por la Comisión Nacional de Bioética (Conbioetica). El acuerdo señala que los CHB tienen como objetivo mejorar los servicios de atención a la salud de los usuarios e implementar políticas que: 1) influyan directamente en la atención de los pacientes; 2) fomenten el respeto a su dignidad y 3 ) reconozcan el derecho que tienen para participar en su tratamiento. Las disposiciones generales cuarta y quinta señalan que dichos comités deberán atender los dilemas (nomenclatura adoptada en México, aunque sería más correcto hablar de "problemas" (Gracia, 2001) éticos suscitados durante la atención de la salud buscando -a través del análisis laico, plural, autónomo e incluyentesalvaguardar la dignidad, los derechos, la seguridad y el bienestar de todos los participantes. En la quinta disposición de este acuerdo se indica que los cHB deben actuar teniendo en cuenta el interés de los participantes en la prestación de los servicios hospitalarios, desarrollando actividades que promuevan la educación bioética permanente y permitan a los miembros del cHB y al personal de salud incorporar información, conocimiento y conductas que faciliten la identificación -y potencialmente la resolución- de los problemas bioéticos a los que se enfrenten. Adicionalmente, esta misma disposición indica explícitamente que uno de los propósitos de los CHB es promover la educación bioética de las comunidades involucradas en la prestación de los servicios.

Discutir el papel de los CHB en materia de educación en salud puede ser muy amplio en la medida en que, por un lado - y debido a la propia naturaleza de la reflexión bioética- esta educación no puede limitarse a la educación médica continua convencional y, por otro lado, los documentos que establecen los objetivos y definen las funciones de los $\mathrm{CHB}$ se refieren a este campo de manera 
muy general. En consecuencia, para desarrollar el tema primero se presentan elementos que permiten ubicar el sentido de las afirmaciones finales.

\section{La educación en salud en los establecimientos hospitalarios}

En primer lugar, se describe brevemente lo que podría denominarse el enfoque clásico de la educación en salud, como se imparte actualmente en el ámbito hospitalario, a fin de analizar las posibilidades que este tipo de educación ofrece para la enseñanza de la bioética como actividad cotidiana en estos establecimientos.

La primera pregunta sería: ¿qué es lo que se enseña? No se refiere a qué problemas de salud, qué temas o qué campos de especialidad son más comúnmente abordados durante la educación en salud; lo que parece más importante es señalar que los contenidos de la educación médica por lo general se presentan tan verdaderos y tan definitivos que parecen poco menos que indudables. De hecho, pareciera que los únicos cambios legítimos de este tipo de saber son aquellos que los hacen más precisos o más profundos. Desde este enfoque, lo que importa es identificar cuáles son los conocimientos verdaderos y las acciones correctas, y la forma como es más económico, efectivo o eficiente llevarlas a cabo. Como es claro, la naturaleza de la bioética impide basar su enseñanza desde este enfoque, en el cuál cada problema solo tiene una respuesta correcta-basada en el conocimiento verdadero- $y$ una sola conducta adecuada.

La segunda pregunta sería: ¿quién educa? Desde la perspectiva clásica o tradicional, el educador es habitualmente el profesional sanitario, el profesional de la salud, casi siempre el médico o la enfermera. Adicionalmente, la creciente complejidad de las ciencias médicas ha determinado que los programas de enseñanza descansen fundamentalmente en especialistas, idealmente con formación en educación para la salud. No obstante, aunque la participación de estos profesionales en la enseñanza de la bioética puede resultar muy relevante, es indispensable ampliar este espectro para el caso de los CHB. La educación para la salud que los CHB son responsables de promover y conducir requiere el concurso de una variedad de profesionales -algunos provenientes del campo de las humanidades y las ciencias sociales-, cuyos intereses no pueden limitarse el ámbito hospitalario.

Con respecto a cómo se educa, hasta hace unos años era habitual que la formación pedagógica de los profesionales de la salud fuera muy poca o nula, por lo que desde hace algunas décadas se promovió la capacitación sistemática en habilidades educativas y pedagógicas que les permitieran transmitir con mayor eficacia el conocimiento. No obstante, debido a que la educación en bioética no consiste fundamentalmente en la trasmisión de conocimientos precisos y técnicas correctas, pues implica la 
transformación de las personas que participan en el proceso educativo, los comités están obligados a desarrollar nuevos y complejos métodos de enseñanza, en ocasiones opuestos a los que se usan en la educación para la salud tradicional.

Al preguntarse por quién se educa, debe aceptarse que es la parte más tradicional de la enseñanza médica, pues por lo general se dirige exclusivamente al personal responsable de prestar la atención y casi siempre de forma escalonada; es decir, impartiendo cursos progresivamente más complejos a personal cada vez más especializado. Por esta razón, los cursos que se imparten en los hospitales se dirigen en primer término a estudiantes, llevando a cabo lo que se conoce como formación de recursos humanos, en pregrado y posgrado. En segundo término se dirigen al personal responsable del servicio, la enseñanza y la investigación, fundamentalmente clínica (lo que se conoce como educación médica continua). Aunque más recientemente la educación para la salud ha incorporado a los profesionales sanitarios de otras ciencias de la salud, es innegable que sus propósitos se dirigen fundamentalmente a mejorar las acciones realizadas en los consultorios, además de las hospitalarias y quirúrgicas. En otras palabras, las acciones educativas en los hospitales buscan mejorar las labores realizadas por los médicos con sus pacientes y ocasionalmente con sus familiares. La educación solo a veces se destina a los familiares y casi nunca a las poblaciones sanas.

Es importante tener estos antecedentes como telón de fondo pues ahora existe la novedad -en la historia de la medicina es una auténtica novedad, considerando una tradición de 2500 años a partir de Hipócrates- de que en los hospitales deben existir $\mathrm{CHB}$. El origen de este tipo de comités se sitúa hacia los años sesenta del siglo $\mathrm{xx}$.

\section{Los comités hospitalarios de bioética y su función educativa}

Las funciones de los chB han sido tres: la educativa, la consultiva y la normativa (Spickery Kushner, 1989; Tealdi, 1995; Gonsoulin y Taube, 2001). En este trabajo solamente se mencionan algunos aspectos de la función normativa y se enfoca hacia la función educativa.

La primera función educativa de los CHB se refiere a la que estos deben proporcionar a sus miembros (Christensen, 1989). Esto es fundamental porque cuando se forma un comité resulta muy común que no todos sus miembros posean capacitación formal en bioética y el propio comité debe proveerles de los conocimientos y habilidades básicos para funcionar apropiadamente. Como generalmente se trata de personas apasionadas por el conocimiento bioético, de alguna forma es relativamente fácil orientar el aprendizaje y homogeneizar sus conocimientos. Los documentos normativos señalan que los miembros 
del comité deben tener algún tipo de formación, la cual puede partir, en principio, del propio comité.

Lo que constituye un verdadero reto es capacitar a los integrantes para debatir en un clima de libertad, tolerancia y respeto. Esta es una tarea que -a diferencia de la enseñanza médica convencional- no consiste esencialmente en el aprendizaje de nuevos conceptos, categorías o modelos, sino que requiere el desarrollo de una forma especial de razonamiento y de una conciencia esencialmente incluyente entre los integrantes de los comités.

Además, los CHB tienen bajo su responsabilidad la promoción de la educación bioética del resto del personal sanitario de la institución (Barlotta y Scheirton, 1989), en especial de aquel que está en contacto cotidiano con los pacientes y sus familiares. Esto resulta fundamental por varios aspectos. El primero es que a todo profesional sanitario se le presentan problemas éticos dentro de la práctica, de manera cotidiana. Afortunadamente, los profesionales cuentan con recursos para resolver la mayoría de estos problemas (gran parte de las veces conseguidos, desgraciadamente, de una manera no formal, es decir, no explícitamente dentro de su formación universitaria y hospitalaria). El segundo aspecto es que hay áreas que suelen concentrar más problemas éticos (el inicio de la vida humana con los obstetras, el final de la vida humana con los intensivistas y paliativistas; los ámbitos de la psiquiatría, pediatría, etc.); es razonable, por ejemplo, que las áreas de mayor problemática sean abordadas primero. Un tercer aspecto es que en ocasiones se llega a casos límite, similares a los mostrados en la literatura especializada, donde el profesional sanitario tiene muchas dudas respecto a cómo resolverlos, ya no desde el punto de vista técnico-clínico, sino ético. Aristóteles menciona en la Ética a Nicómaco que, en circunstancias difíciles, hay que preguntar a los amigos. En el marco contemporáneo, el CHB se constituye precisamente para que le pregunten.

El último punto es el de la educación hacia la comunidad (Fox et al., 1989). La "comunidad" considera no solo a pacientes y familiares, sino también a la población que acude realmente a la unidad hospitalaria y que incluso podría ampliarse a la población que potencialmente podría acudir.

La forma como estos comités han aparecido en la normatividad nacional mexicana es como cHB. Tratando de encontrar las primeras referencias a esta nomenclatura, pudimos ver que se encuentran en el título de la revista canónica sobre el tema (Health Care Ethics Committee Forum: An Interprofessional Journal on Healthcare Institutions' Ethical and Legal Issues, publicación de la casa editorial Springer desde 1989). Aunque la nomenclatura no es uniforme internacionalmente (también se denominan "comités de ética clínica", "comités institucionales de ética", etc.), la mayor parte de los aspectos de contenido es muy estable, lo que confirma las tres funciones mencionadas. 
En el caso de la función educativa y a pesar de que no existen muchos documentos académicos, en general se acepta que los comités -como señala la quinta disposición general del acuerdo para su integración y funcionamiento en México-deben desarrollar actividades que permitan a sus miembros y al personal de salud del establecimiento incorporar información, conocimiento y conductas para identificar y resolver dilemas bioéticos. Esta disposición es totalmente acorde a las funciones educativas tradicionalmente desarrolladas en los establecimientos de salud, ya señalados.

En el acuerdo por el que se emiten las "Disposiciones Generales para la Integración y Funcionamiento de los Comités Hospitalarios de Bioética" y se establecen las unidades hospitalarias que deben contar con ellos, de conformidad con los criterios establecidos por la Conbioetica, publicado en el Dof el 31 de octubre de 2012, hay tres secciones donde explícitamente se habla de las funciones educativas del comité. La sección quinta dice que:

Las funciones del comité serán: [...] V. Desarrollar un conjunto de actividades que ayuden a los miembros del Comité y al personal de salud del Establecimiento a incorporar información, conocimiento y conductas para la identificación y posible resolución de Dilemas bioéticos y así promover la educación bioética permanente de sus miembros y del personal del establecimiento. (s. p.)

La sección décima establece que:

El Presidente podrá convocar a sesionar al Comité, a petición que se le haga por escrito, del personal de atención médica, de un paciente o su familiar directo, tutor o representante legal, que reciba la atención en salud dentro del Establecimiento y así como por los involucrados en el proceso de educación para la salud [...]. (s. p.)

Finalmente, la sección décimo tercera consigna que: "El Comité promoverá con el director o titular del Establecimiento, la elaboración de lineamientos y guías institucionales para la atención y la docencia médica. Así mismo, promoverá la educación bioética permanente de sus miembros y del personal del Establecimiento" (s. p.).

La sección décima del acuerdo se indica: "el presidente podrá convocar a sesionar el comité a petición que se le haga por escrito del personal de atención médica, de un paciente o su familiar directo" (s. p.). Esta posibilidad de petición -que puede variar según la normativa de cada país- en México incluye tanto a quienes directamente reciben atención dentro del establecimiento como a todos aquellos "[...] involucrados en el proceso de atención [...]". Desde este punto de vista, cualquier persona involucrada en un posible problema bioético puede solicitar atención por parte del снв. 
Esto incluye los problemas presentados durante la educación para la salud. La sección xiII señala además que "[...] el comité promoverá [...] la elaboración de lineamientos y guías institucionales para la atención y la docencia médica" (s. p.). Esto significa que la función normativa del comité también debe ejercerse en materia educativa. Todo problema que enfrente el personal de salud como parte de una intervención educativa y que potencialmente implique un componente ético es también problema del cHB.

A pesar de lo anterior, debe anotarse que una cosa es mencionar las funciones educativas del comité y su relación con las funciones consultiva y normativa, y otra es indicar qué es lo que se va a enseñar, a quién se va a educar, quién va a educar y cómo se va a educar. Es decir, responder a las preguntas planteadas al inicio del trabajo y que, en el caso de la educación en bioética, implican una ruptura con las formas educativas que convencionalmente se han desarrollado en los hospitales.

\section{Los comités hospitalarios de bioética y el contenido de la educación en salud}

La Unesco ha desarrollado tres guías relacionadas con los comités de bioética. La guía 2 se titula Funcionamiento de los comités de bioética: procedimientos y políticas; al tratar el tema del funcionamiento de los comités, propone temáticas respecto de la labor educativa de estos. En la sección 4, que se refiere a "Qué necesitan saber los comités de bioética: Preparación de futuros presidentes y miembros" (p. 59), se proponen dos bloques conceptuales. El primero recomienda "Temas generales de bioética para miembros de los comités (autonomía individual, derecho, justicia, inocuidad, beneficencia)", y en la siguiente sección se mencionan "Temas de bioética para miembros de los comités", donde se sugiere que "Los comités de ética asistencial se centrarán en cuestiones como el mejoramiento de la atención del paciente, decisiones de limitación del soporte vital y biotecnologías reproductivas [...]" (p. 60).

Estos temas deben ampliarse y adaptarse a cada realidad. Por ejemplo, en México la mayoría de los centros hospitalarios no tienen acceso a biotecnologías reproductivas, por lo que los problemas más frecuentes se referirán seguramente a otros aspectos, Lo que permite la normatividad actual es ampliar el marco de ejercicio de los $\mathrm{CHB}$, de manera que sea posible atender las múltiples problemáticas éticas que puede presentar la atención hospitalaria en los diferentes contextos particulares.

La cuestión más relevante entonces es delimitar cuáles podrían ser los temas que los CHB podrían enfrentar más frecuentemente en materia de educación para la salud, y muy en especial los que competen a la educación de la comunidad. Ya se ha señalado el papel de estos comités en la educación del personal 
del hospital y en la formación bioética del resto de los involucrados. Para cualquiera de los casos (ya sea personal de salud, pacientes, familiares o población sana), pueden elaborarse listas de temas o aspectos relacionados con la problemática más frecuentemente enfrentada en el terreno bioético. Una primera lista se refiere a los problemas relacionados con el final de la vida humana (tabla 1).

La voluntad anticipada, por ejemplo, es un tema de interés nacional y debería ser analizado regularmente por los CHB. Algunos temas muy actuales, como la muerte digna, no se encuentran explícitamente planteados entre los retos señalados por la normatividad para los CHB, pero quizás deberían hacerse explícitos en el seno de los comités.

En otras materias importantes, como en la eutanasia, la LET, la sedación paliativa o el suicidio medicamente asistido, aún hace falta realizar amplios debates sociales, tanto entre la comunidad científica como entre la población general, pacientes y familiares, que finalmente son las poblaciones afectadas. Estos temas deberían ser promovidos y dirigidos por los Снв.

Al inicio de la vida humana también existe otra serie de cuestiones que deberían ser evaluadas como campo de educación en salud (tabla 2). En este caso se encuentran la sexualidad y la reproducción.

Tabla 1. Problemas éticos relacionados con el final de la vida humana.

\begin{tabular}{lll}
$\begin{array}{l}\text { Problemaséticos relacionadoscon } \\
\text { la actuación del personal sanitario }\end{array}$ & $\begin{array}{l}\text { Problemas éticos que se pre- } \\
\text { sentan por solicitudes de pa- } \\
\text { cientes }\end{array}$ & $\begin{array}{l}\text { Problemas éticos para abor- } \\
\text { darse y deliberar a nivel co- } \\
\text { munitario }\end{array}$ \\
$\begin{array}{l}\text { Limitación del esfuerzo terapéu- } \\
\text { tico (let). }\end{array}$ & Eutanasia & Muerte digna. \\
$\begin{array}{l}\text { Cuidados paliativos y sedación } \\
\text { paliativa. }\end{array}$ & Suicidio médicamente asistido. & Voluntades anticipadas. \\
\hline
\end{tabular}

Fuente: elaboración propia.

Tabla 2. Problemas éticos relacionados con el inicio de la vida humana

\begin{tabular}{ll}
\hline Problemas éticos relacionados con el erotismo & $\begin{array}{l}\text { Problemas éticos relacionados con la repro- } \\
\text { ducción }\end{array}$ \\
Diversidad sexual. & $\begin{array}{l}\text { Técnicas de reproducción humana asistida. } \\
\text { Aborto, interrupción voluntaria del embarazo } \\
\text { (ive), e interrupción legal del embarazo (ile). } \\
\text { Homofobia. }\end{array}$ \\
\hline
\end{tabular}

Fuente: elaboración propia. 
Existen, cuando menos, un par de temas que probablemente estarían entre los más difíciles de tratar desde el punto de vista educativo. La dificultad no reside en el terreno teórico, y parte del hecho de que la mayoría de las personas no tiene formación específica para tratar cuestiones relacionadas con la sexualidad humana, pues para tratar temas de sexualidad es necesario tener un proceso de capacitación específico en el área.

Cuando se habla de IVE, se limita por lo general a los embarazos de hasta doce semanas, que es el límite referido en la literatura convencional. Pero, ¿qué sucede cuando una usuaria tiene la necesidad de interrumpir su embarazo después de esta semana? Estos asuntos tendrían que dialogarse en la comunidad en materia de educación. Igual sucede con la cuestión de la imagenología y lo que se debería hacer ante un producto con malformaciones.

Con respecto a la interrupción del embarazo, en primer término, es necesario indicar que el aborto y la IVE son dos entidades distintas, aunque comúnmente se les considere lo mismo. Una cosa es cuando una mujer de manera espontánea pierde su embarazo -lo que tiene connotaciones técnicas y éticas de cierta naturaleza-y otra cuando una mujer por voluntad propia-independientemente de las causas que la llevaron a tomar tal decisión-decide interrumpir su embarazo. Si bien el resultado parece el mismo (el fin de un embarazo), se trata de dos cuestiones éticas y técnicas totalmente diferentes. En Ciudad de México este tema es de mayor importancia, ya que la legislación vigente ha despenalizado la IVE y existe un programa de iLE. Desde el punto de vista filosófico, la voluntariedad es lo que le proporciona la característica de ético al fenómeno. Pero, como bien se sabe, lo legal no siempre es sinónimo de ético ni lo ético es sinónimo de legal. Parece claro que la reflexión sobre la IVE se desarrollará de una manera distinta según se trate de un contexto de legalidad o de ilegalidad. Esta es la razón por la que debe considerarse la problemática que cada comité podría enfrentar en su práctica diaria, de acuerdo con su particular contexto (o el caso de las comisiones estatales para la organización adoptada en México). Por ejemplo, la discusión sobre IVE y la educación para la salud respectiva no serán las mismas en el estado de Guanajuato -donde pueden encontrarse testimonios de mujeres que han sido encarceladas por haber presentado un aborto espontáneo (Redacción de Proceso, 2010)- que en Ciudad de México. Si un CHB considera importante establecer un programa de educación en la comunidad que tenga en cuenta la posibilidad de una IVE, y el cual tendría que informar a las mujeres que desean interrumpir su embarazo que pueden acudir a una de las instituciones del Distrito Federal donde es posible hacerlo sin penalización legal. Hasta este momento, solo el 2 \% de las usuarias del programa de ile reside fuera de Ciudad de México, aunque el programa permite usuarias que no radiquen en esta entidad; pero 
la población no lo sabe. Sería uno de los tópicos que podrían discutir los $\mathrm{CHB}$ respecto a la educación en estos temas. En este aspecto hay que ser muy claros: debe resaltarse que es diferente educary promover. A nadie se debe invitar a que interrumpa un embarazo si no quiere; esta es una cuestión importantísima en materia de educación. De hecho, las mujeres que voluntariamente interrumpen su embarazo siempre se ven obligadas a hacerlo. Que una mujer interrumpa su embarazo es una verdadera desgracia y ante esta desgracia hay que tomar una serie de medidas desde el punto de vista médico y otras desde el punto de vista ético. Este es uno de los temas que podría incorporar la agenda de los CHB.

Otro grave problema es el conformado por la discriminación sexual y la homofobia, presentes todavía en los servicios de salud. Un primer problema -uno de los más graves- se refiere a la "normalización" del discurso homofóbico entre los propios profesionales. Recientemente la Suprema Corte de Justicia de la Nación ha resuelto que términos como maricón y puñal son discriminatorios y propios del discurso homofóbico, y que a pesar de su uso común y arraigado en lengua española (aparecen en el Diccionario de la Real Academia Española), su utilización viola los derechos humanos de los afectados. También es innegable que todavía se niega la atención a personas con preferencias sexuales distintas a las consideradas "normales", o que esta atención es de más baja calidad que la que se brinda a los heterosexuales. Ante los atentados a la diversidad sexual en favor de una supuesta "normalidad", debe reafirmarse que, en el fondo, la norma es la diversidad. No puede combatirse, en cuanto profesionales, un problema tan severo como la homofobia si no se aborda como un asunto que involucra a todo personal de salud. No obstante, debe reconocerse que no es fácil abordar el tema de la educación sexual y menos aún en la comunidad, pues ocurre que generalmente lo que se hace es transmitir los propios prejuicios y creencias propias, a falta de una educación formal en el tema.

\section{Los comités hospitalarios de bioética y la educación en el Estado contemporáneo}

Adela Cortina (1986, 2008), autora ampliamente reconocida en el ámbito de la filosofía moral y política, parte de una realidad en el "mundo occidental": se vive en democracias liberales, al menos desde el punto de vista formal, con todas las matizaciones que puedan formularse. A final de cuentas en México, Latinoamérica, Europa, se vive en democracias liberales.

Se ha tratado previamente el tema de la deliberación, ¿cuál será el éxito de los procesos de deliberación en los $\mathrm{CHB}$ ? Aún no se sabe, falta experiencia para poder exponer algo sobre el tema. Pero es preocupante que la mayoría de las veces se hable de "deliberar", de "dialogar", y no se profundiza en ello; pareciera que se asume que todo 
el mundo puede hacerlo. En realidad, todos pueden opinar, pero la bioética no es el mundo de la mera opinión: entre la opinión y la argumentación media un largo proceso de lectura y reflexión. No todos los argumentos valen igual, y se trata de buscar los mejores. Una de las grandes dificultades personales para poder deliberar es la creencia, que tiene la mayoría de los seres humanos, de que los mejores argumentos son los propios. Por ello, lo que intentan muchos es ir a un comité a convencer a los demás; esto no lleva a ningún lado. Si los integrantes del comité no tienen claro que los mejores argumentos pueden no ser los propios, no podrán deliberar (Gracia, 2004; Gracia, 2006).

Cortina propone un marco sobre lo que significa vivir en una democracia liberal. Esta autora recapitula los dos grandes tópicos que ha manejado la ética a lo largo de 2500 años de evolución: cuestiones de justicia y de felicidad. En una sociedad como la propia de las democracias liberales, deberían respetarse unos mínimos de justicia compartidos por toda la población. Esto es lo que Cortina llama una "ética de mínimos", cuya función sería hacer posible que la justicia sea la misma y valga igual para todos. Tras este proceso de articulación social, en el cual todos deberían estar de acuerdo en qué es lo justo, parece prudente recodar el caso de la IVE. Si una mujer cursa por una desgracia personal que la lleva a interrumpir su embarazo, parece ser que no debería ser penalizada bajo ciertos mínimos de justicia compartida. Si casi una cuarta parte de la población mexicana vive en Ciudad de México, habría que ver si ese mínimo de justicia — que consiste en no penalizar a estas mujeres- puede irse ampliando en el espacio geográfico y, si no se amplía (pues hay que recordar que otras variables, de tipo político, tienen un peso importante en estas decisiones), debe tratarse de permitir, por lo menos, el acceso de la población a estas opciones a partir de la movilidad que resulta de los procesos de educación.

¿Por qué resulta fundamental contar con estas bases mínimas de justicia? Porque, a nivel individual, todos los seres humanos tienen ideales de vida buena, de vida feliz, de vida en plenitud. Desde Aristóteles se afirma que todos los seres humanos tienden a la felicidad: todo ser humano quiere una vida feliz, y estos proyectos se determinan por cada quien. Si bien la justicia es algo bastante exigente y exigible, la felicidad es algo que jamás se puede exigir, que jamás se puede imponer y a la que solamente se puede invitar. Si una persona pertenece $\mathrm{a}$ un determinado credo secular o religioso, y cree realmente que en eso consiste "ser bueno", puede invitar a quien quiera, pero nunca debe imponerlo, como tampoco permitir que se le imponga el credo de otro. Esto es lo que Cortina (1986) llama las "éticas de máximos", que corresponde a los máximos de vida buena, que cada quien determina para sí mismo. Si una mujer embarazada no quiere interrumpir su embarazo porque le parece que no se 
ajusta a su proyecto de vida buena, que es un pecado o que no le lleva a la plenitud, debe respetarse su decisión. Otra mujer embarazada, que por alguna desgracia de la vida requiere interrumpir su embarazo, tiene el mismo derecho a tomar su propia decisión.

Elaborar argumentos para sostener estas posiciones no es sencillo. Cortina (2008) plantea que en las sociedades con democracia deliberativa, el éxito de articular una ética cívica de mínimos con varias éticas de máximos depende de varios factores y uno de ellos es la generación de una ciudadanía compleja. ¿Qué quiere decir ciudadanía compleja?, evidentemente, lo contrario de una ciudadanía simple. ¿A qué se refiere la ciudadanía simple?, a la construcción de una ciudadanía prescindiendo de las diferencias. Por lo menos desde el mundo moderno se tiene la idea de que todos los seres humanos son iguales en dignidad. Desde luego, fue un gran paso respecto del mundo feudal, pero esta consideración de igualdad se realiza prescindiendo de muchas cualidades: existen varones y mujeres, heterosexuales y homosexuales, mujeres que quieren interrumpir su embarazo y mujeres que no lo quieren, etc. Resulta ser que todos son iguales en dignidad, pero prescindiendo de las diferencias y como en realidad no se puede prescindir de las diferencias - porque todos los seres humanos son diferentes-, lo que es un verdadero reto es construir ciudadanía a partir de las diferencias. Tan difícil es y tanto ha costado en Occidente, que para el caso de México es hasta una modificación constitucional en 2001, en la cual se explicita el derecho a no ser discriminados por ser diferentes; la norma, en realidad, es que los seres humanos son diferentes.

La construcción de esta ciudadanía compleja se articula con un segundo punto, el contar con una sociedad plural. Las sociedades son plurales desde todos los puntos de vista: son plurales desde el punto de vista político porque unos votan a la izquierda, otros a la derecha y otros no votan; son plurales desde el punto de vista religioso, porque unos pertenecen a una determinada religión y otros no pertenecen a ninguna religión, unos son católicos, otros judíos, musulmanes, agnósticos, ateos, etc. Las sociedades son plurales, pero no son laicas. Decir que una sociedad es laica es un error, aunque pueda encontrarse tal afirmación en la literatura sobre el tema; las sociedades no son laicas, las sociedades son plurales.

¿Qué es lo que debe ser laico? Se trata del tercer elemento que plantea Cortina (2008), que es el Estado. El Estado tiene que ser laico, porque en una sociedad plural lo que corresponde para poder realizar los proyectos personales de vida buena y de vida feliz, desde un marco mínimo de justicia compartida, es un Estado laico. Cortina hace una clasificación y considera que los Estados pueden ser clasificados en tres categorías distintas. La primera es la de los Estados confesionales, aquellos que consideran 
que su poder político emana de alguna religión. Puede invocarse al Medio Oriente, a los Estados teocráticos, pero es alejarse de las democracias liberales. En occidente existe un estado teocrático por excelencia: el Estado Vaticano. Desde sus supuestos teológicos, es Dios quien elige a su representante en la tierra, por intermedio de los obispos; Dios le da el poder político a ese Estado. En América, Costa Rica es un Estado confesional porque en su constitución dice, en el artículo 75, que "La Religión católica apostólica romana es la del Estado, el cual contribuye a su mantenimiento".

El principal problema del Estado confesional es que desde su configuración como Estado exige dividir a los ciudadanos en dos grupos: los ciudadanos "de primera" y los ciudadanos "de segunda". Los primeros son aquellos que creen en la religión oficial, y los segundos son los que no creen en ella. Los ciudadanos de segunda están siempre en una mala posición respecto de los ciudadanos de primera. En el caso de Costa Rica, por ejemplo, hay que recordar que interrumpir un embarazo es siempre ilegal (incluso en los casos de violación) y que fue el único país no islámico que declaró anticonstitucional la reproducción humana asistida. Las mujeres que no crean en la religión de Estado están, evidentemente, en una mala situación.

El segundo tipo de Estado es el laicista, un tipo de Estado que piensa que la vivencia de la religiosidad es fuente de mal para los ciudadanos por generar desigualdad entre ellos y entonces se preocupa por erradicar las religiones. En el Estado laicista el problema se invierte y no se gana mucho, porque los ciudadanos de primera son los que no creen y los de segunda son los que creen.

Sin embargo, las democracias liberales presumen que todos los ciudadanos deben ser tratados con igual consideración y respeto. Si esto es así, entonces el Estado no puede ni debe introducir diferencias entre ciudadanos de primera y ciudadanos de segunda, por lo que no puede ser confesional ni laicista, sino laico. Debe respetar ciertos mínimos de justicia para que cada quien crea lo que le parezca mejor para sus proyectos individuales de vida buena, de vida feliz.

\section{Conclusiones}

Finalmente, es necesario preguntarse si es posible este nuevo panorama educativo para los Снв. Desde luego va a demandar mucho más que lo que se ha manejado sobre la función consultiva y la función normativa, ya que esto abre un panorama verdaderamente novedoso respecto a la calidad y cantidad de los contenidos que debe incluir el comité. Los profesionales de la salud tienen 2500 años siendo "perros viejos" con una larga tradición; por eso parece que costará trabajo introducir todos estos factores educativos en los CHB (Tweeddale, 2001), pero debe hacerse gradualmente, por el beneficio de las acciones relacionadas con la salud comunitaria, con la salud colectiva, con la salud de la población. 
Una gran pregunta es si toda esta labor tendrá impacto en la práctica clínica. Una investigación analiza los marcos nacionales de los Estados Unidos, el Reino Unido y Francia — que han sido los ejemplos de lo que puede hacerse en materia de comités de ética y regulaciones nacionales. Los autores concluyen que dependerá del marco institucional nacional, cómo se desarrolle y cómo se aplique finalmente la actividad de los comités (Gaucher et al., 2013). Si la literatura especializada propone a los CHB como instituciones plurales, laicas, tolerantes e incluyentes, parece que su labor puede tener un alcance mucho más allá del ámbito hospitalario, rebasando el ámbito clínico y logrando un impacto colectivo. Esto implica la necesidad de desarrollar, desde un mínimo compartido de justicia, una ética de máximos personales, capaz de impulsar los proyectos individuales de vida buena y feliz.

En este marco general, la labor de los CHB puede ser fundamental para lograr que cada quien, respetando ciertos mínimos de justicia, pueda — desde sus personales visiones de vida buena - realizarse en la bondad moral que le corresponda.

\section{Referencias}

Barlotta, F. M. y Scheirton, L. S. (1989). The role of the hospital ethics committee in educating members of the medical staff. Hospital Ethics Committees Forum, 1(3), 151-158.
Christensen, K. T. (1989). Self-education for hospital ethics committees. Hospital Ethics Committees Forum, 1(6), 333-339.

Cortina, A. (1986). Ética minima. Madrid: Tecnos.

Cortina, A. (2008). Lo justo como núcleo de las ciencias morales y politicas. Una versión cordial de la ética del discurso. Madrid: Real Academia de Ciencias Morales y Políticas.

Diario Oficial de la Federación [dof]. (14 de diciembre de 2011). Decreto por el que se adiciona el artículo 41 Bis y se reforma el artículo 98 de la Ley General de Salud. Recuperado de http://dof.gob.mx/ nota_detalle.php?codigo $=5224260 \& f e-$ cha $=14 / 12 / 2011$

Diario Oficial de la Federación [dof]. (31 de octubre 2012). Acuerdo por el que se emiten las Disposiciones Generales para la Integración y Funcionamiento de los Comités Hospitalarios de Bioética y se establecen las unidades hospitalarias que deben contar con ellos, de conformidad con los criterios establecidos por la Comisión Nacional de Bioética. Recuperado de http://dof.gob.mx/nota_detalle.php?codigo $=5276107 \&$ \&echa $=31 / 10 / 2012$

Fox, R. A., Terry, P. B. y Gottlieb, L. (1989). Should hospital ethics committees provide community education? Hospital Ethics Committees Forum, 1(3), 159-162.

Gaucher, N., Lantos, J. y Payot, A. (2013). How do national guidelines frame clinical ethics practice? A comparative analysis of guidelines from the US, the UK, Canada and France. Social Science E Medicine, 85, 74-78.

Gonsoulin, T. P. y Taube, J. M. (2002). Hospital ethics committees: formation, 
function, and case consultation. Journal of the Louisiana State Medical Society, 154(6), 323-327.

Gracia, D. (2001). Moral deliberation: The role of methodologies in clinical ethics. Medicine, Health Care and Philosophy, 4(2), 223-232.

Gracia, D. (2006). Contribución de las humanidades médicas a la formación del médico. Humanitas. Humanidades Médicas, 1. Recuperado de http://www. iatros.es/wp-content/uploads/humanitas/materiales/TM1.pdf

Gracia, D. (2004). Como arqueros al blanco. Estudios de Bioética. Madrid: Triacastela.

Redacción de Proceso. (2010). Guanajuato: Criminalización de la mujer. Recuperado el 10 de octubre de 2016, de http://www. proceso.com.mx/82111/82111-guanajuato-criminalizacion-de-la-mujer

Spicker, S. F. y Kushner, T. (1989). The principal functions of HECs. Hospital Ethics Committees Forum, 1(2), 57-62.

Tealdi, J. C. (1995). Los comités hospitalarios de ética, seis años después. Cuadernos del Programa Regional de Bioética, 1(1), 129-144.

Tweeddale, M. G. (2001). Teaching old dogs new tricks-a personal perspective on a decade of efforts by a clinical ethics committee to promote awareness of medical ethics. Journal of Medical Ethics, 27(Suppl. 1), i41-i43. 